\title{
Claudin-1 is a strong prognostic indicator in stage II colonic cancer: a tissue microarray study
}

\author{
Murray B Resnick ${ }^{1}$, Tamako Konkin ${ }^{1}$, Justin Routhier ${ }^{1}$, Edmond Sabo ${ }^{2}$ and Victor E Pricolo ${ }^{3}$ \\ ${ }^{1}$ Department of Pathology, Rhode Island Hospital and Brown University School of Medicine, Providence, \\ RI, USA; ${ }^{2}$ Department of Pathology, Carmel Medical Center, Haifa, Israel and ${ }^{3}$ Department of Surgery, \\ Rhode Island Hospital and Brown University School of Medicine, Providence, RI, USA
}

\begin{abstract}
Tight junction associated proteins are key molecular components governing cellular adhesion, polarity and glandular differentiation. Tight junction proteins also play critical roles in cellular proliferation and neoplastic pathways via their functions as couplers of the extracellular milieu to intracellular signaling pathways and the cytoskeleton. Neoplastic cells frequently exhibit structural and functional deficiencies in the tight junction. The purpose of this study was to determine the pattern of expression and prognostic value of four tight junction associated proteins, claudin-1, claudin-4, occludin and ZO-1 in a cohort of TNM stage II colon cancer using tissue microarray technology. In this study, we retrospectively analyzed, resected and otherwise untreated paraffin embedded specimens from 129 consecutive patients with TNM stage II colonic carcinomas for claudin1, claudin-4, occludin and ZO-1 protein expression by immunohistochemistry. Seventy-five, 58,56 and $44 \%$ of the tumors exhibited normal to elevated expression levels ( +2 and +3 immunopositivity) of claudin-1, claudin4, occludin and ZO-1 respectively. Low expression levels of claudin-1 and ZO-1 were directly associated with higher tumor grade $(P=0.05$ and 0.03 respectively). Multivariate analysis indicated that lymphovascular invasion $(P=0.01)$ and low levels of claudin-1 $(P=0.0001)$ expression were independent predictors of recurrence and that reduced claudin-1 expression $(P=0.0001)$ was associated with poor survival. This study is the first to comprehensively examine the expression of several tight junction associated proteins in colonic neoplasms and to correlate their expression with disease progression. Loss of claudin-1 expression proved to be a strong predictor of disease recurrence and poor patient survival in stage II colon cancer.
\end{abstract}

Modern Pathology (2005) 18, 511-518, advance online publication, 8 October 2004; doi:10.1038/modpathol.3800301

Keywords: claudin-1; claudin-4; occludin; prognostic factor; tight junction; ZO-1

A hallmark of cancer is loss of cellular organization. The histological grade of colorectal carcinomas is an important prognostic variable and depends on the degree of glandular differentiation and cellular polarity. High-grade, poorly differentiated colorectal neoplasms are usually more aggressive than their low-grade, well-differentiated counterparts. ${ }^{1}$ One of the key molecular components governing cellular adhesion and polarity is the tight junction. ${ }^{2} \mathrm{Neo}-$ plastic cells frequently exhibit structural and functional deficiencies in the tight junction. ${ }^{3-6}$ In normal epithelia, the primary functions of the tight junction are to seal the apical intercellular spaces of

Correspondence: Dr MB Resnick, MD, PhD, Department of Pathology, Rhode Island Hospital, 593 Eddy Street, Providence, RI 02903, USA.

E-mail: mresnick@lifespan.org

Received 22 June 2004; revised 27 August 2004; accepted 28

August 2004; published online 8 October 2004 glandular epithelia creating a barrier against the paracellular passage of macromolecules and to separate the plasma membrane into the apical and basolateral domains. ${ }^{7,8}$ It is hypothesized that tight junction proteins play critical roles in the neoplastic process via their roles as couplers of the extracellular milieu to intracellular signaling pathways and the cytoskeleton. ${ }^{9,10}$ Alterations in tight junction permeability may also allow increased diffusion of nutrients and other factors critical for tumor growth and survival. ${ }^{11}$ In addition, loss of tight junction integrity may be an important step in the development of the metastatic phenotype. ${ }^{12}$

The tight junction is comprised of integral proteins such as occludin, ${ }^{13}$ and the claudin family of proteins, ${ }^{14}$ as well as tight junction-associated proteins such as ZO-1. ${ }^{15}$ Occludin is expressed ubiquitously in tight junctions and has been shown to contribute directly to tight junction function, whereas ZO-1 is thought to regulate occludin 
function. ${ }^{13,16}$ There are currently at least 24 known members of the claudin family, which are expressed in a tissue-specific pattern. ${ }^{17,18}$ Claudins bind to each other via two extracellular loops, ${ }^{17}$ and also bind to ZO-1 via their carboxyl terminus. ${ }^{19} \mathrm{ZO}-1$ in turn binds the tight junction to the actin cytoskeleton and interacts with several cell signaling and transcriptional regulatory proteins..$^{9,10,15}$ The characteristics of tight junctions differ in epithelia of various organs. These differences are related to different ratios of expression between the tight junction proteins in general as well as those of claudin family specifically.

Relatively few studies have examined the role of tight junction proteins in the development of colorectal neoplasia. Occludin and ZO-1 are downregulated in poorly differentiated colorectal adenocarcinomas, ${ }^{20-22}$ whereas only one report has studied the level of claudin expression in colonic tumors. ${ }^{23}$ To the best of our knowledge, no studies have correlated the expression of any of the tight junction proteins with patient survival in colorectal cancer, although one report has linked reduced ZO-1 expression and liver metastases. ${ }^{21}$

In this study, we examine the expression of several members of the tight junction family; ZO-1, occludin, claudin-1 and claudin-4 in tissue microarrays constructed from a cohort of stage II colon cancer patients. Tissue microarrays are especially suitable for the immunohistochemical examination of multiple markers. ${ }^{24}$ The simultaneous evaluation of multiple cases on each slide virtually eliminates slide-to-slide variation, which is an important factor contributing to variability in immunohistochemical studies. The expression levels of these tight junction proteins are correlated with each other, tumor grade, disease recurrence and patient survival.

\section{Materials and methods}

\section{Patients and Specimens}

Archival cases of TNM stage II colon cancer (not including rectal cancer) from 129 consecutive patients were retrieved nonselectively from the archives of the Department of Pathology at Rhode Island Hospital between the years of 1983 and 1994. Stage was defined according to American Joint Committee on Cancer criteria. ${ }^{25}$ None of these patients received adjuvant chemotherapy or radiotherapy before surgery or after the initial resection. Recurrence and survival data were ascertained through the Rhode Island Tumor Registry. This study was approved by the Institutional Review Board at Rhode Island Hospital.

All tissue samples were formalin-fixed and paraffin-embedded. The corresponding H\&E slides were reviewed for confirmation of diagnosis and adequacy of material by MR. Each case was classified by the pathologist (MR) according to grade (high or low), ${ }^{25}$ mucinous differentiation (more than $50 \%$ of the tumor cells), and presence or absence of lymphovascular invasion.

\section{Tissue Microarray Construction}

Paraffin blocks containing areas consisting of pure invasive carcinoma were identified on corresponding $\mathrm{H} \& \mathrm{E}$-stained sections. Areas of interest which represented the 'invasive front' of the tumor, rich in non-necrotic tumoral glands, were identified and marked on the source block. The source block was cored and a $1 \mathrm{~mm}$ core transferred to the recipient 'master block' using the Beecher Tissue Microarrayer (Beecher Instruments, Silver Spring, MD, USA). Three to six cores were arrayed per specimen.

\section{Immunohistochemistry}

Immunohistochemistry for each antigen (claudin-1, claudin-4, occludin and ZO-1) was performed on $5 \mu \mathrm{m}$ paraffin sections of each colon cancer tissue microarray section described above. Slides were stained with claudin-1 (polyclonal rabbit, 1:125, Zymed Laboratories South San Francisco, CA, USA) and claudin-4 (monoclonal mouse clone 3E2C1, 1500, Zymed Laboratories) using the Vector ABC Elite Kit (Vector Laboratories, Burlingame, CA, USA). Briefly, following deparrafinization, antigen retrieval was performed in $10 \mathrm{mM}$ citrate buffer using a microwave/pressure cooker. Following $\mathrm{H}_{2} \mathrm{O}_{2}$ and serum blocking, slides were incubated with the primary antibody at room temperature overnight. The secondary antibody was either a goat anti-rabbit IgG biotin (1:500, Vector Laboratories) or horse antimouse IgG biotin (1:500, Vector Laboratories), respectively. Detection was performed with the DAB kit (Vector Laboratories). Staining for occludin (polyclonal rabbit, 1:125, Zymed Laboratories) and ZO-1 (polyclonal rabbit, 1:250, Zymed Laboratories) was performed using the DAKO, DAB Envision kit (DAKO, Copenhagen, Denmark). Protease pretreatment at $37^{\circ} \mathrm{C}$ for 5 min was performed before $\mathrm{H}_{2} \mathrm{O}_{2}$ blocking.

Slides were counterstained with hematoxylin, dehydrated, cleared and mounted. Positive controls consisted of normal colonic mucosa. Negative controls included replacement of the primary antibody with nonreacting antibodies of the same species.

\section{Immunohistochemistry Assessment}

For scoring purposes, the intensity of staining of claudin-1- and claudin-4-expressing neoplastic cells was compared to that of normal colonic mucosa. Tumors that displayed a well-localized linear circumferential membranous staining pattern equal to that of normal colonic mucosa were scored as +2 . Tumors exhibiting a more intense staining pattern 
than that of normal colonic mucosa were scored as +3 , whereas tumors exhibiting a considerably lower intensity than normal mucosa were scored as +1 . The scoring of the intensity of occludin and ZO-1 staining was performed similarly by comparing the staining of the neoplastic cells to the linear/ punctate pattern of staining seen at the apical surface of the normal colonic epithelium. The extent of staining (percentage of cells staining) was, for the most part, homogenous within each $1 \mathrm{~mm}$ core and, therefore, was not included as a variable.

At least three cores were scored per case. The analysis of three cores per case has been shown to be comparable to the analysis of the whole section in a recent study ${ }^{26}$ and in a pilot validation study in our lab. The vast majority of cases exhibited a uniform degree of staining between all cores and in those that did not, an average score was determined. All sections were scored independently by MR without knowledge of the clinicopathological features or clinical outcome.

\section{Statistical Analysis}

For statistical evaluations, the immunohistochemical staining intensities were grouped as low (which included scores 0 and +1 ) and normal to elevated (which included scores +2 and +3 ). Associations between binary categories were analyzed using the $\chi^{2}$ test. The influence of prognostic factors on tumor-related recurrence as well as survival was assessed by Kaplan-Meier estimates, and subgroups were compared by the log-rank test for univariate analysis.

The multivariate Cox's proportional hazard model was applied using a stepwise forward method in order to detect independent predictors of recurrence/survival. Two-tailed $P$-values of 0.05 or less were considered to be statistically significant.

\section{Results}

\section{Clinicopathological Features (Table 1)}

The mean age of the patients at initial surgery was 72.5 years (range, 31-96 years), 60 males and 69 females were included in this study. The mean duration of follow-up was 96 months. Of the tumors, 61 were right-sided and 68 were left-sided with the reference point being the splenic flexure. No rectal or rectosigmoid cases were included in this study. The degree of tumor differentiation as defined by the AJCC $^{25}$ was as follows: 18 high grade (poorly differentiated); 111 low grade (well differentiated); 26 mucinous; 103 nonmucinous. Evidence of lymphatic or vascular invasion was present in 11 cases.

The status of the patients and incidence of recurrent disease is also illustrated in Table 1. In all, 22 patients had recurrent disease (seven local and 15 distal metastases). A total of 92 patients died
Table 1 Clinicopathological characteristics of 129 colonic cancer patients

\begin{tabular}{|c|c|}
\hline Variable & $\mathrm{n}$ \\
\hline \multicolumn{2}{|l|}{ Age at surgery (years) } \\
\hline Mean & $72.5 \pm 10.0$ \\
\hline Range & 31-96 \\
\hline \multicolumn{2}{|l|}{ Sex } \\
\hline Male & 60 \\
\hline Female & 69 \\
\hline \multicolumn{2}{|l|}{ Tumor Location ${ }^{\mathrm{a}}$} \\
\hline Proximal & 61 \\
\hline Distal & 68 \\
\hline \multicolumn{2}{|l|}{ Differentiation } \\
\hline High grade & 18 \\
\hline Low grade & 111 \\
\hline Non-mucinous & 104 \\
\hline Mucinous & 25 \\
\hline \multicolumn{2}{|l|}{ Lympho-vascular invasion } \\
\hline Present & 11 \\
\hline Absent & 118 \\
\hline \multicolumn{2}{|l|}{ Colon cancer relapse } \\
\hline None & 94 \\
\hline Local & 7 \\
\hline Distal & 15 \\
\hline Information unavailable & 13 \\
\hline \multicolumn{2}{|l|}{ Vital statistics } \\
\hline Alive & 37 \\
\hline Dead_all causes & 92 \\
\hline Dead-unrelated & 55 \\
\hline Dead-colon cancer & 21 \\
\hline Information unavailable & 16 \\
\hline
\end{tabular}

${ }^{\mathrm{a}}$ Reference point being the splenic flexure.

during the follow-up period, 21 died of disease, and 55 of unrelated causes, whereas the cause of death for 16 patients was unclear.

\section{Pattern of Expression of Tight Junction Proteins in Colonic Neoplasms}

Normal colonic epithelium exhibited a circumferential membranous pattern of staining for claudin-1 and claudin-4. The immunohistochemical staining pattern of the colonic tumors for claudin-1 and claudin-4 was similar to that seen for the normal colonic mucosa, namely a circumferential membranous pattern of staining (Figure 1a,b). A few tumors exhibited a weak cytoplasmic pattern of staining for the claudins. When present this was either weaker or equal to the concurrent membranous pattern. A more fragmented and weaker circumferential pattern of staining for these proteins was seen primarily in poorly differentiated tumors.

In optimally sectioned, normal colonic mucosa, ZO-1 and occludin were expressed at the apical border of the colonic epithelium in a linear pattern with a dot-like accentuation at the apical adhesion 

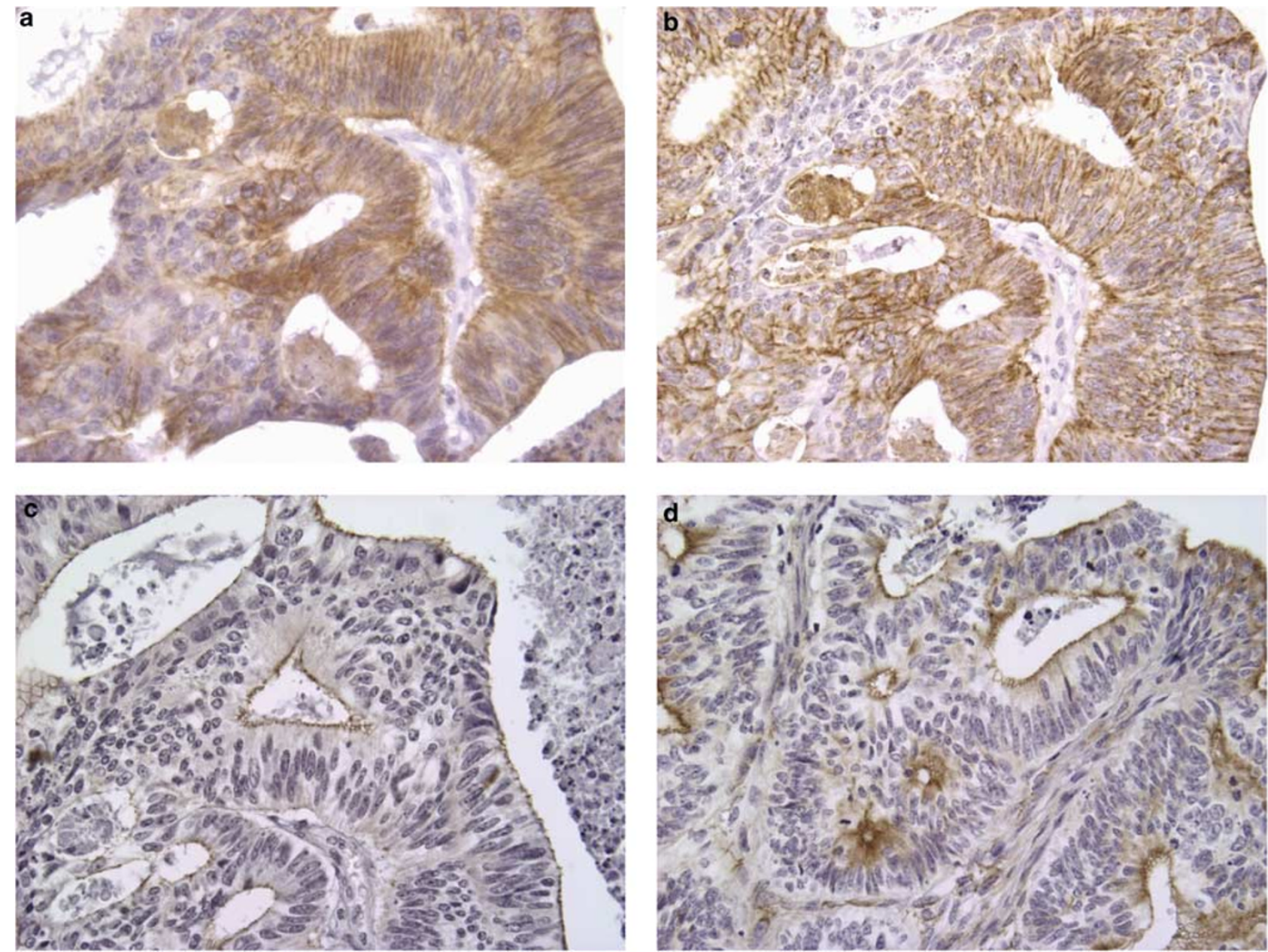

Figure 1 Claudin-1, claudin-4, occludin and ZO-1 expression in representative colonic tumors on a tissue microarray. (a) Strong, diffuse, membranous staining for claudin-1. (b) Strong, diffuse membranous staining for claudin-4. (c) Apical linear staining pattern for occludin. (d) Apical linear staining pattern for ZO-1. All figures are at $\times 400$.

site. In the well-differentiated gland-forming tumors occludin and ZO-1 were variably expressed at the apical cell border (Figure 1c, d). No tumors exhibited a cytoplasmic staining pattern for occludin and two poorly differentiated tumors stained for ZO-1 in a weakly cytoplasmic pattern. A subset of poorly differentiated carcinomas exhibited a moderate to strong, segmental linear to dot-like membranous pattern of staining for occludin while a smaller subset exhibited a similar pattern for ZO-1. This staining pattern was accentuated around primitive lumina and at lateral cellular junctions (Figure 2).

\section{Pattern of Expression of Tight Junction Proteins in Colonic Neoplasms}

The frequency of expression of the tight junctions evaluated is described in Table 2 . The vast majority of the colonic cancers exhibited some degree of staining for claudin-1, claudin-4 and occludin (98, 97 and $95 \%$, respectively), whereas $21 \%$ of the tumors were negative for ZO-1. When stratifying the frequency of expression into two groups-low

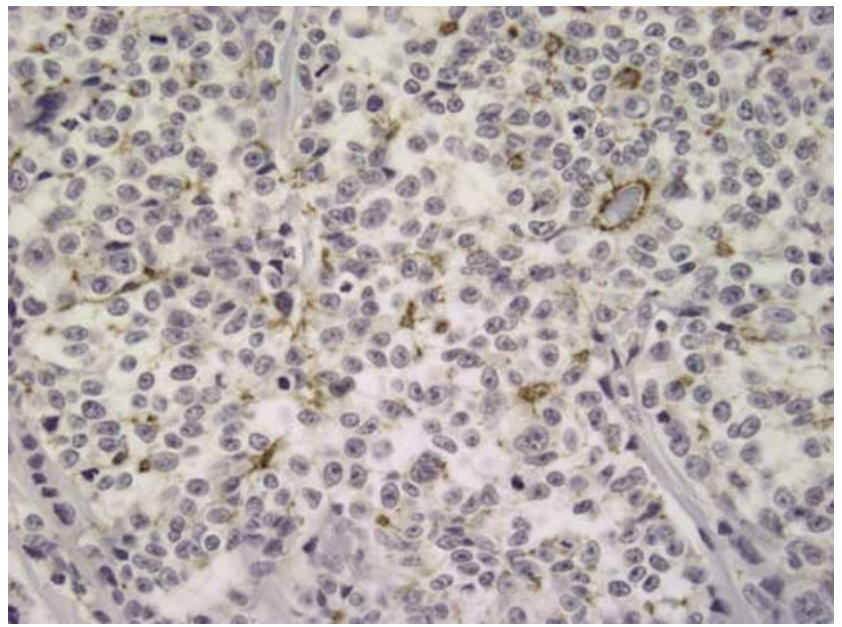

Figure 2 Positive dot-like to segmental, membranous occludin staining with accentuation around primitive glands in poorly differentiated colon cancer $(\times 400)$.

( 0 and +1 immunopositivity) and normal to elevated ( +2 and +3 immunopositivity), 76, 58, 56 and $44 \%$ of the tumors exhibited normal to elevated expres- 
sion levels of claudin-1, claudin-4, occludin and ZO-1, respectively.

\section{Correlation between Tight Junction Protein Expression and Other Clinicopathological Features}

As shown in Table 3, levels of claudin-1 and ZO-1 expression were inversely correlated with tumor grade $(P=0.05$ and 0.03 , respectively). A strong inverse trend was detected between levels of occludin expression and tumor grade $(P=0.06)$. No significant correlation was detected between the expression of any of the proteins studied with mucinous differentiation or lymphovascular invasion.

Univariate analysis of recurrence (log-rank) (Table 4) revealed that high tumor grade $(P=0.008)$, lymphovascular invasion $(P=0.03)$ and low levels of claudin-1 expression $(P=<0.0001)$ were predictors of recurrence. Univariate analysis of survival (log-rank) (Table 4) revealed that reduced claudin-1 expression $(P=<0.0001)$ was associated with poor survival, whereas a strong trend $(P=0.08)$ was found between tumor grade and survival.

Table 2 Frequency of different levels of tight junction protein expression

\begin{tabular}{lcccccc}
\hline & $\begin{array}{c}1+ \\
\mathrm{n}(\%)\end{array}$ & $\mathrm{n}(\%)$ & $\mathrm{n}(\%)$ & $\mathrm{n}(\%)$ & $\mathrm{n}(\%)$ & $\mathrm{n}(\%)$ \\
\hline Claudin-1 & $2(2)$ & $30(23)$ & $45(35)$ & $51(40)$ & $32(34)$ & $97(76)$ \\
Claudin-4 & $4(3)$ & $49(39)$ & $44(34)$ & $30(24)$ & $30(24)$ & $74(58)$ \\
Occludin & $7(5)$ & $49(39)$ & $55(43)$ & $16(13)$ & $56(44)$ & $71(56)$ \\
ZO-1 & $27(21)$ & $44(34)$ & $35(27)$ & $22(17)$ & $71(56)$ & $57(44)$ \\
\hline
\end{tabular}

${ }^{\mathrm{a}} \mathrm{L}$ (absent/low, 0 or $1+$ staining), $\mathrm{H}$ (normal/elevated, 2 or $3+$ staining).

Table 3 Association between tight junction protein expression and tumor grade

\begin{tabular}{lccc}
\hline & \multicolumn{2}{c}{ Tumor differentiation } & \\
\cline { 2 - 3 } & Well & Poorly & P-value* \\
& & & \\
\hline $\begin{array}{l}\text { Claudin-1 } \\
\quad \text { Absent/low }\end{array}$ & 23 & 8 & 0.051 \\
$\quad$ Normal/elevated & 85 & 11 & \\
& & & \\
Claudin-4 & 41 & 11 & 0.11 \\
$\quad$ Absent/low & 66 & 8 & \\
Normal/elevated & & & \\
Occludin & & 12 & 0.062 \\
$\quad$ Absent/low & 43 & 7 & \\
Normal/elevated & 64 & & \\
ZO-1 & & 15 & 0.028 \\
Absent/low & 56 & 4 & \\
$\quad$ Normal/elevated & 52 & & \\
\hline
\end{tabular}

${ }^{\mathrm{a}}$ Absent/low (0 or $1+$ staining), normal/elevated (2+ or $3+$ staining).

${ }^{*} P$-value $\left(\chi^{2}\right.$ test $)$
Table 4 Univariate analysis of recurrence and survival

\begin{tabular}{lcc}
\hline Variable & $\begin{array}{c}\text { Recurrence } \\
\text { P (log-rank's) }\end{array}$ & $\begin{array}{c}\text { Survival } \\
\text { P (log-rank's) }\end{array}$ \\
\hline Grade & $0.008^{\mathrm{b}}$ & 0.08 \\
LVI $^{\mathrm{a}}$ & $0.03^{\mathrm{b}}$ & 0.22 \\
Claudin-1 & $<0.0001^{\mathrm{b}}$ & $<0.0001^{\mathrm{b}}$ \\
Claudin-4 & 0.23 & 0.12 \\
Occludin & 0.10 & 0.24 \\
ZO-1 & 0.35 & 0.49 \\
\hline
\end{tabular}

${ }^{\mathrm{a}}$ Lymphatic or vascular invasion.

${ }^{\mathrm{b}}$ Significant.

Multivariate Cox analysis revealed that lymphovascular invasion and reduced claudin-1 staining were associated with recurrent disease, $P=0.01$ and 0.0001 , respectively, whereas only reduced claudin-1 expression correlated with poor survival $(P=0.0001)$. Kaplan-Meier plots illustrating the association of claudin-1 expression with recurrence and survival are shown in Figure $3 a$ and $b$.

\section{Discussion}

Much is known regarding the function of tight junction proteins in the maintenance of normal glandular epithelial physiology; however, their role in the neoplastic process is less well defined. Relatively few studies have examined the expression pattern of claudins in human neoplasia in general or in colonic cancer specifically. To the best of our knowledge this is the first study which correlates claudin protein expression with disease recurrence or patient survival in any neoplasm. Claudin-1 mRNA levels have been shown to be reduced in breast cancer, ${ }^{27}$ and claudin-23 levels downregulated in intestinal-type gastric cancer. $^{28}$ Similar to our study, loss of claudin-7 protein expression correlated with histological grade in invasive ductal carcinoma; ${ }^{29}$ however, no correlation was detected between loss of claudin-7 protein expression and tumor stage or lymph-node metastases.

Only one report has examined the expression of claudins in colorectal neoplasia. ${ }^{23}$ In a study limited to 16 colorectal cancers, Miwa et $a l^{23}$ reported that claudin-1 is frequently upregulated in neoplastic tissue as compared to normal mucosa. The degree of differentiation of the tumors in this study was not described nor was the prognostic significance addressed. Similar to Miwa's study, the majority of tumors in our study (75\%), exhibited claudin-1 protein expression levels equal or higher to that seen in the adjacent normal colonic mucosa. However, here we show that reduced claudin-1 expression correlated with poor differentiation, and perhaps more importantly, strongly correlated with disease recurrence and poor patient survival. Moreover, in our study loss of claudin-1 expression was a 
516

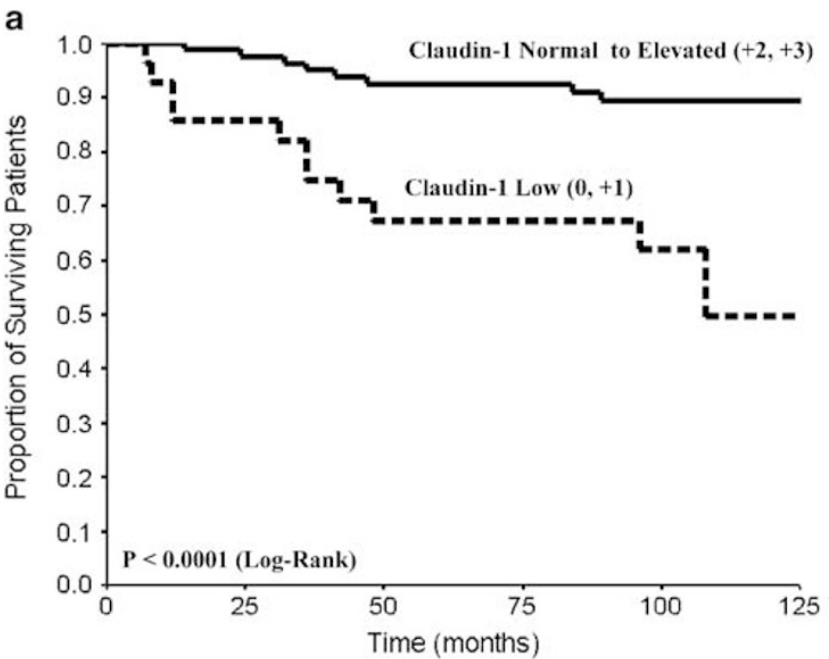

b

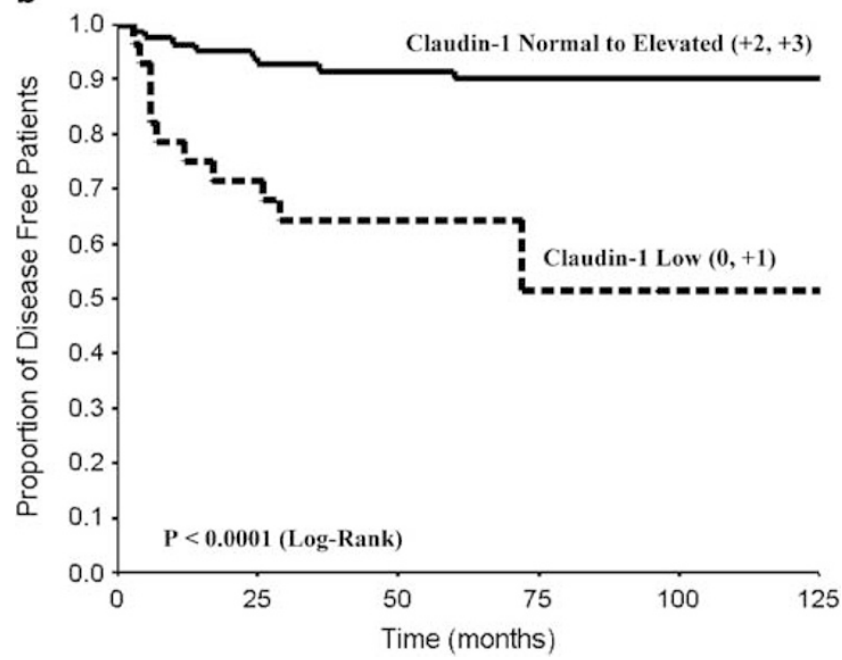

Figure 3 Kaplan-Meier disease-free and survival analysis curves in colonic cancer for claudin-1 expression. (a) A strong association was detected between low expression levels of claudin-1 ( 0 , +1 ) and disease recurrence. (b) A strong association was detected between low expression levels of claudin- $1(0,+1)$ and patient survival.

stronger predictor of recurrence and survival than either lymphovascular invasion or grade.

In this study, loss of claudin-4 expression was not associated with differentiation or prognosis. Paradoxically, levels of claudin-4 have been shown to be upregulated in pancreatic ductal carcinoma ${ }^{30,31}$ and in ovarian cancer. ${ }^{32,33}$ In vitro studies suggest that claudin-4 overexpression leads to decreased invasiveness and metastatic potential in pancreatic cancer. ${ }^{34}$ Similar although less striking than claudin-1, the level of claudin-4 expression in the colonic tumors we studied was equal or stronger than the adjacent colonic mucosa in $58 \%$ of the tumors. It is not clear why reduced expression of claudin-1, but not claudin-4, correlated significantly with poor prognosis, however, this finding suggests that loss of certain claudins have greater impact on tumor aggression than others. This is not surprising as claudin proteins are differentially expressed in different tissues. ${ }^{18}$ The fact that claudin-7 but not claudin-1, 3 and 4 is lost in higher grade breast cancers further supports this observation. ${ }^{29}$

The effect of claudin loss on neoplastic progression via tight junction dismantling is easy to comprehend. It has also recently been shown that claudin-1 reexpression in vitro leads to apoptosis in breast cancer spheroids suggesting yet another mechanism whereby claudin loss may lead to tumor progression. ${ }^{35}$ However, it is not quite as clear how upregulation of claudins may contribute to neoplastic progression. It is possible that upregulation or aberrant expression of a certain claudin may alter normal tight junction structure contributing to the neoplastic process. In support of this theory, Furuse et $a l^{36}$ have shown that upregulation of claudin-2 in MDCK cells leads to decreased tight junction function. Based on our findings it can be hypothesized that increased claudin-1 expression may play a contributory role to the neoplastic process of welldifferentiated colonic tumors; however, loss of claudin-1 expression contributes to a poorly differentiated phenotype as well as to disease progression.

Few studies have examined the expression of occluding or ZO-1 in colorectal cancer. ${ }^{20-22}$ Tobioka et $a^{22}$ determined that the expression of occludin, and Kimura et $a l^{20}$ demonstrated that expression of both occludin and ZO-1 were decreased in solid, poorly differentiated areas of colorectal cancer. These two studies did not address the prognostic significance of ZO-1 or occludin loss. In a more recent report, Kaihara et $a l^{21}$ examined the expression of ZO-1 and e-cadherin in colorectal cancer and found that their expression levels were inversely correlated with tumor differentiation and with metastases.

In this study, we also demonstrate that ZO-1 expression is inversely correlated with tumor grade whereas a strong trend was found between reduced occludin expression and poorly differentiated tumors. This observation may be related to the fact that more poorly differentiated tumors exhibited moderate to strong expression for occludin (7/19), than for ZO-1 (4/19). These poorly differentiated tumors exhibited a segmental, linear to dot-like membranous pattern of staining, which was accentuated around primitive lumina and at lateral cellular junctions. Kleeff et $a l^{37}$ also described a similar pattern of ZO-1 expression in poorly differentiated pancreatic cancer. The biological significance of this distribution pattern of occludin (or ZO-1) in poorly differentiated tumors is unclear.

One mechanism whereby tight junction proteins may be involved in neoplastic progression is through coupling of the extracellular milieu to intracellular signaling pathways and the cytoskeleton. Of all the tight junction-associated proteins examined here, the interactions of $\mathrm{ZO}-1$ with proteins associated with the neoplastic process are probably the best characterized. ZO-1 interacts with 
several signaling proteins such as the RAS substrate AF-6, ${ }^{38,39}$ G-proteins ${ }^{40}$ and connexin $43 .{ }^{41}$ It has also been hypothesized that an intact tight junction protein complex may suppress tumorogenesis. In support of this hypothesis, Willott et $a l^{42}$ reported that ZO-1 is homologous to the discs-large tumor suppressor protein $\mathrm{dlg}$ of Drosophila. Mutation of dlg leads to loss of epithelial polarity and to the development of neoplasia. Dlg has also been shown to bind with the adenomatous polyposis coli gene product whose role as a tumor suppressor gene in colonic cancer is well defined. ${ }^{43} \mathrm{ZO}-1$ also binds $\beta$ catenin. ${ }^{44}$ Loss of ZO-1 may lead to transfer of $\beta$ catenin to the nucleus where it has been shown to function as a transcription factor. ${ }^{45}$ Finally, activation of EGF-R, which is commonly seen in colorectal cancer, leads to tyrosine phosphorylation of ZO-1. ${ }^{46}$ It has been suggested that phosphorylation of ZO-1 leads to downregulation of function and glandular dedifferentiation. ${ }^{46}$

The expression level of claudin-1 may prove to be a useful prognostic marker for colon cancer in general and stage II colon cancer specifically. This is especially critical for determining which stage II colon cancer patients should receive adjuvant therapy. Despite the fact that $60-75 \%$ of patients with stage II colon cancer are cured with surgery alone and most patients are not treated with adjuvant chemotherapy, some patients may receive chemotherapy unnecessarily while others, who may benefit from it, are not treated. ${ }^{47}$

This study is the first to examine comprehensively the expression of several tight junctionassociated proteins in colonic neoplasms and to correlate their expression with disease progression in a cohort of stage II colonic cancer patients. Reduced expression of claudin-1 and ZO-1 was significantly associated with poorly differentiated tumors. Loss of claudin-1 expression was a strong predictor of disease recurrence and poor patient survival in stage II colon cancer and we propose its use as a novel prognostic factor in colonic cancer.

\section{Acknowledgements}

We acknowledge the clerical assistance of Laura Gantt and Dr Ronald DeLellis for critical evaluation of the manuscript. This research was supported by the Center for Cancer Research Development (COBRE)-Molecular Pathology, National Center for Research Resources \#5 P20 RR017695-02 and The Radiation Therapy Oncology Group (RTOG), RTOG Seed Grant Research Program \#CA21661.

\section{References}

1 Hermanek P, Sobin LH. Colorectal carcinoma. In: Hermanek P, Gospodarowicz MK, Henson DE (eds). Prognostic Factors in Cancer. Springer-Verlag: New York, 1995, pp 64-79.
2 Tsukita S, Furuse M, Itoh M. Multifunctional strands in tight junctions. Nat Rev Mol Cell Biol 2001;2: 285-293.

3 Weinstein RS, Mer FB, Alroy J. The structure and function of intercellular junctions in cancer. Adv Cancer Res 1976;23:23-89.

4 Kerjaschki D, Krisch K, Sleyter UB, et al. The structure of tight junctions in human thyroid tumors. A freezefracture study. Am J Pathol 1979;96:207-225.

5 Polak-Charcon S, Shoham J, Ben-Shaul Y. Tight junctions in epithelial cells of human fetal hindgut, normal colon and colon adenocarcinoma. J Natl Cancer Inst 1980;65:53-62.

6 Soler AP, Miller RD, Laughlin KV, et al. Increased tight junction permeability is associated with the development of colon cancer. Carcinogenesis (Lond), 1999;20: 1425-1431.

7 Gumbiner B, Louvard D. Localized barriers in the plasma membrane: a common way to form domains. Trends Biochem Sci 1985;10:435-438.

8 Madara JL. Regulation of the movement of solutes across tight junctions. Annu Rev Physiol 1998;60: 143-159.

9 Mitic LL, Andersen JM. Molecular architecture of tight junctions. Annu Rev Physiol 1998;60:121-142.

10 Balda MS, Matter K. The tight junction protein ZO-1 and an interacting transcription factor regulate erb-2 expression. Eur Mol Biol Org 2000;19:2024-2033.

11 Mullin JM. Potential interplay between luminal growth factors and increased tight junction permeability in epithelial carcinogenesis. J Exp Zool 1997; 279:484-489.

12 Ren J, Hamada J, Takeichi N, et al. Ultrastructural differences in junctional intercellular communication between highly and weakly metastatic clones derived from rat mammary carcinoma. Cancer Res 1990;50: 358-362.

13 Furuse M, Itoh M, Hirase T, et al. Direct association of occludin with ZO-1 and its possible involvement in the localization of occludin at the tight junction. J Cell Biol 1994;127:1617-1626.

14 Furuse M, Fujita K, Hiiragi T, et al. Claudin-1 and -2: novel integral membrane proteins localizing at tight junctions with no sequence similarity to occludin. J Cell Biol 1998;141:1539-1550.

15 Fanning AS, Jameson BJ, Jesaitis LA, et al. The tight junction protein ZO-1 establishes a link between the transmembrane protein occludin and the actin cytoskeleton. J Biol Chem 1998;273:29745-29753.

16 McCarthy KM, Skare IB, Stankewich MC, et al. Occludin is a functional component of the tight junction. J Cell Sci 1996;109:2287-2298.

17 Morita K, Furuse M, Fujimoto K, et al. Claudin multigene family encoding four-transmembrane domain protein components of tight junction strands. Proc Natl Acad Sci USA 1999;96:511-516.

18 Tsukita S, Furuse M, Itoh M. Multifunctional strands in tight junctions. Nat Rev Mol Cell Bio 2001;2:285-293.

19 Itoh M, Furuse M, Morita K, et al. Direct binding of three tight junction-associated MAGUKs, ZO-1, ZO-2, and $\mathrm{ZO}-3$ with the $\mathrm{COOH}$ termini of claudins. J Cell Biol 1999;147:1351-1363.

20 Kimura Y, Shiozaki H, Hirao M, et al. Expression of occludin tight junction associated protein in human digestive tract. Am J Pathol 1997;151:45-54.

21 Kaihara T, Kusaka T, Nishi M, et al. Redifferentiation and ZO-1 re-expression in liver metastasized colorectal 
cancer: possible association with epidermal growth factor receptor induced tyrosine phosphorylation of ZO-1. Cancer Sci 2003;166-172.

22 Tobioka H, Isomura H, Kokai Y, et al. Polarized distribution of carcinoembryonic antigen is associated with a tight junction molecule in human colorectal adenocarcinoma. J Pathol 2002;198:207-212.

23 Miwa N, Furuse M, Tsukita S, et al. Involvement of claudin-1 in the b-catenin/Tcf signaling pathway and its frequent upregulation in human colorectal cancers. Oncol Res 2001;12:469-476.

24 Kononen J, Bubendorf L, Kallioniemi A, et al. Tissue microarrays for high-throughput molecular profiling of tumor specimens. Nat Med 1998;4:844-847.

25 Greene FL, Page DL, Fleming ID, et al (eds). In: AJCC: Cancer Staging Handbook: From the AJCC Cancer Staging Manual, 6th edn. Springer-Verlag: New York, 2002, pp 127-129.

26 Jourdan F, Sebbagh N, Comperat E, et al. Tissue microarray technology: validation in colorectal carcinoma and analysis of p53, hMLH1, and hMSH2 immunohistochemical expression. Virchows Arch 2003;443:115-121.

27 Kramer F, White K, Kubbies M, et al. Genomic organization of claudin-1 and its assessment in hereditary and sporadic breast cancer. Hum Genet 2000;107:249-256.

28 Katoh M, Katoh M. CLDN 23 gene, frequently downregulated in intestinal-type gastric cancer, is a novel member of CLAUDIN gene family. Int J Mol Med 2003;11:683-689.

29 Kominsky SL, Argani P, Korz D, et al. Loss of the tight junction protein claudin-7 correlates with histological grade in both ductal carcinoma in situ and invasive ductal carcinoma of the breast. Oncogene 2003;22: 2021-2033.

30 Sato N, Fukushima N, Maitra A, et al. Gene expression profiling identifies genes associated with invasive intraductal papillary mucinous neoplasms of the pancreas. Am J Pathol 2004;164:903-914.

31 Nichols LS, Ashfaq R, Iacobuzio-Donahue CA. Claudin 4 expression in primary and metastatic pancreatic cancer: support for use as a therapeutic target. Am J Clin Path 2004;121:226-230.

32 Hough CD, Sherman-Baust CA, Pizer ES, et al. Largescale serial analysis of gene expression reveals genes differentially expressed in ovarian cancer. Cancer Res 2000;60:6281-6287.

33 Rangel LB, Agarwal R, D’Souza T, et al. Tight junction proteins claudin-3 and claudin- 4 are frequently overexpressed in ovarian cancer but not in ovarian cystadenomas. Clin Cancer Res 2003;9:2567-2575.
34 Michl P, Barth C, Buchholz M, et al. Claudin-4 expression decreases invasiveness and metastatic potential of pancreatic cancer. Cancer Res 2003;63: 6265-6271.

35 Hoevel T, Macek R, Swisshelm K, et al. Reexpression of the TJ protein claudin-1 induces apoptosis in breast cancer spheroids. Int J Cancer 2004;108:374-383.

36 Furuse M, Furuse K, Sasaki H, et al. Conversion of zonulae occludens from tight to leaky strand type by introducing claudin-2 into Madin-Darby canine kidney I cells. J Cell Biol 2001;153:263-272.

37 Kleeff J, Shi X, Bode HP, et al. Altered expression and localization of the tight junction protein $\mathrm{ZO}-1$ in primary and metastatic pancreatic cancer. Pancreas 2001;23:259-265.

38 Yamamoto T, Harada N, Kano K, et al. The Ras target AF-6 interacts with ZO-1 and serves as a peripheral component of tight junctions in epithelial cells. J Cell Biol 1997;139:785-795.

39 Yamamoto $\mathrm{T}$, Harada $\mathrm{N}$, Kawano $\mathrm{Y}$, et al. In vivo interaction of AF-6 with activated Ras and ZO-1. Biochem Biophys Res Commun 1999;259:103-107.

40 Denker BM, Saha C, Khawaja S, et al. Involvement of heterotrimeric $\mathrm{G}$ protein alpha subunit in tight junction biogenesis. J Biol Chem 1996;271:25750-25753.

41 Toyofuku T, Yabuki M, Otsu K, et al. Direct association of the gap junction protein connexin-43 with ZO-1 in cardiac myocytes. J Biol Chem 1998;273:12725-12731.

42 Willott E, Balda MS, Fanning AS, et al. The tight junction protein ZO-1 is homologous to the Drosophila discs-large tumor suppressor protein of septate junctions. Proc Natl Acad Sci USA 2003;90:7834-7838.

43 Matsumine A, Ogai A, Senda T. Binding of APC to the human homolog of the Drosophila discs large tumor suppressor protein. Science 1996;272:1020-1023.

44 Rajasekaran AK, Hojo M, Huima T, et al. Catenins and zonula occludens-1 form a complex during early stages in the assembly of tight junctions. J Cell Biol 1996; 132:451-463.

45 Morin PJ, Sparks AB, Korinek V, et al. Activation of beta-catenin-Tcf signaling in colon cancer by mutations in beta-catenin or APC. Science 1997;275:1752-1753.

46 Italie CMV, Balda MS, Andersen JM. Epidermal growth factor induces tyrosine phosphorylation and organization of tight junction protein ZO-1 in A431 cells. J Cell Sci 1995;108:1735-1742.

47 Allegra C, Paik S, Colangelo LH, et al. Prognostic value of thymidylate synthase, Ki-67, and p53 in patients with Dukes' B and C colon cancer: A National Cancer Institute-National Surgical Adjuvant Breast and Bowel Project Collaborative Study. J Clin Oncol 2003; 21:241-250. 